\title{
Identifying the Location of a Mobile Object in Real- time using PID-controlled Moving Objects Spatio- Temporal Model
}

\author{
Wang Zhi, Kil Young Sung, and Kyou Ho Lee*, Member, KIMICS
}

\begin{abstract}
Trilateration is a typical method to locate an object, which requires inherently at least three prerecognized reference points. In some cases, owing to out of reachability to communication facilities the target node cannot be reachable always to three base stations. This paper presents a predictive method, which can identify the location of a moving target node in real time even though the target node could not get in touch with all three base stations. The method is based on the PIDcontrolled Moving Objects Spatio-Temporal Model Algorithm. Simulation results verify that this method can predict the moving direction of a moving target, and then combine with its past position information to judge accurately the location.
\end{abstract}

Index Terms - Real Time Locating, Prediction, PID control, Trilateration, Spatio-Temporal Model Algorithm

\section{INTRODUCTION}

IDENTIFYING or tracking the location of a user in real time is essentially required for the u-health service that a user could occasionally be in the situation beyond selfregulation. Real-time locating is usually applicable to mobile objects which communicate with each other and perform metering distances with or without reference to resident systems of coordinates. Trilateration is a generally accepted method to calculate coordinates of the objects, which requires inherently to range from at least three different pre-identified reference points[1]. Such various technologies as ROA(RSSI of Arrival), AOA(Angle of Arrival), TOA(Time of Arrival), TDOA(Time Difference of Arrival), TWR(Two Way Ranging), SDSTWR(Symmetrical Double Sided-Two Way Ranging), etc. have been presented for ranging[2]-[5].

Users, in some cases, become out of reachability in any such reasons as out of propagation ranges, fault of a communication counterpart, non-existence of possible communication facility, etc.[6]-[8] Such cases could not

\footnotetext{
Manuscript received August 14, 2011; revised August 20, 2011; accepted September 1, 2011.

* Corresponding Author

Wang Zhi and Kyou Ho Lee are with the Department of Information and Communications Engineering, Inje University, Gimhae, 621-749, Korea (Email: wangzhi86813@163.com,kyou@inje.ac.kr)

Kil Young Sung is with the Department of Information and Communication Engineering, Gyeongsang National University, Tongyeong, 650-160, Korea (Email: kysung3836@hanmail.net)
}

make user devices, i.e. the object nodes, always reachable to all three base stations. An accession of latest location information, even if insufficient references are given for trilateration, can make it possible to identify the location of the object preciously[9].

This paper presents a method of identifying locations of mobile objects in real-time, which is applicable even to the case that an object node could not get in touch with all three base stations. A predictive method, called PIDcontrolled Moving Objects Spatio-Temporal Model Algorithm, is proposed for the basis of this method. Simulation results not only verify the presented method valid but also the proposed algorithm can perform better than existed ones.

This paper is organized as follow. Section 2 describes the problem of locating a mobile object, which motivates the study of this paper. In Section 3, we propose the PIDcontrolled Moving Objects Spatio-Temporal Model Algorithm. Section 4 justifies the proposed method through simulation analysis. Simulation results also show that the algorithm outperforms comparatively. This paper is concluded in Section5.

\section{PROBLEM OF LOCATING}

\section{A. Locating by Trilateration}

As shown in Fig. 1, the location of the node $A$ is preciously identified by the trilateration method. $R_{l}, R_{2}$ and $R_{3}$ in the figure are three resident reference points, and $d_{1}, d_{2}$ and $d_{3}$ are distances between three resident reference points, $R_{1}, R_{2}$ and $R_{3}$, and the object node $(A)$, respectively, which could be measured by any ranging method described in Section I.

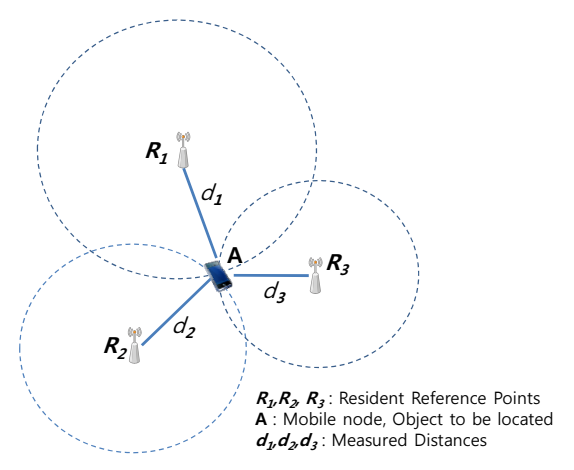

Fig. 1. Location identifying by trilateration 


\section{B. Locating with Partial Reachability}

As described above, the mobile object may not always be reachable those three references. It can be placed in any such cases as out of propagation ranges, fault of a communication counterpart, non-existence of possible communication facility, hardness of constructing resident coordinates, etc. Fig. 2 shows the case that that the object node is reachable to only two resident references. There are two resident references, $R_{l}$ and $R_{2}$, and two ranging information, $d_{1}$ and $d_{2}$, which can be recognized by the object node. Such partial reachability is insufficient for the calculus of trilateration.

In Fig. 2, however, it comes to know that the location of $A$ is either $A_{1}$ or $A_{2}$ by acquiring two range information, $d_{1}$ and $d_{2}$, which are distances of the object node from $R_{l}$ and $R_{2}$ respectively. In addition, the latest location information of the node $A$ can be a clue for getting the moving direction of the node. It is easily to conclude the current location of $A$ between $A_{1}$ and $A_{2}$ by applying such past location data.

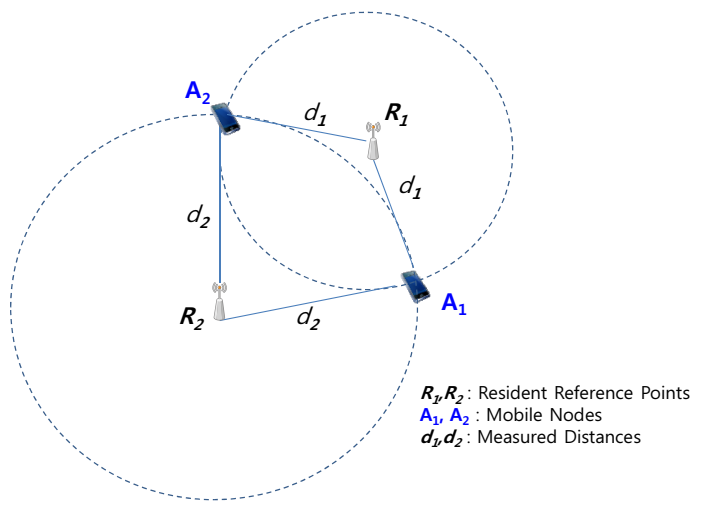

Fig. 2. Identifying by two resident references

The object in the case that only one resident reference is available is located on the circumference of a circle established by the range to the reference. The moving direction of the object is predictive with an accession of its latest location information. The location can be identified by the geometrical cross of the predictive direction and the circumference. The less number of resident references are given, however, the less accurate result should be.

Accuracy in locating with partial reachability depends on how preciously the moving direction is predictive. Thus a prediction algorithm should be significant in that case.

\section{Locating with Mobile Reference}

All references are not required to be resident in real time locating problem. Since calculation of locating only includes relative ranging data between references and the object, a well-identified mobile node, instead of a resident reference, can also be adopted into references to locate another mobile node. Fig. 3 explains such a case.

The node $A$ in Fig. 3, if its location has already been identified with such a manner as given in Fig. 2, can be used as a reference for locating the new target object $B$. We consider four different locations of $B$. In the case of given three references, $R_{1}, R_{2}$ and $A$, the object $B$ could be easily located. Given any two references, either $R_{I}$ and $A$ or $R_{2}$ and $A$, the location of $B$, i.e. either $B_{1}$ or $B_{2}$ respectively in Fig. 3, can be identified with the same manner as locating the node $A$. For the case of $B_{3}$, the target $\operatorname{object}(B)$ is out of reachable ranges of two existing resident references, $R_{I}$ and $R_{2}$. $B$ can only be ranged by the node $A$ with measuring the distance $d_{3}$. It means that the node $B$ is placed on the arc that the circumference of establishing with a radius, $d_{3}$, is not overlapped with any circular area of maximum reachabilities of $R_{l}$ and $R_{2}$, i.e. $d_{1 L}$ and $d_{2 L}$ respectively in Fig. 3.

In any case of $B_{1}, B_{2}$ and $B_{3}$, it comes to estimate its moving direction with an accession of its latest location information. Both such an estimated direction and wellidentified resident and/or mobile references, even if insufficient references are given for trilateration, make it possible to identify the location of the object preciously. As described before, a prediction algorithm should be significant for better accuracy of identification in these cases.

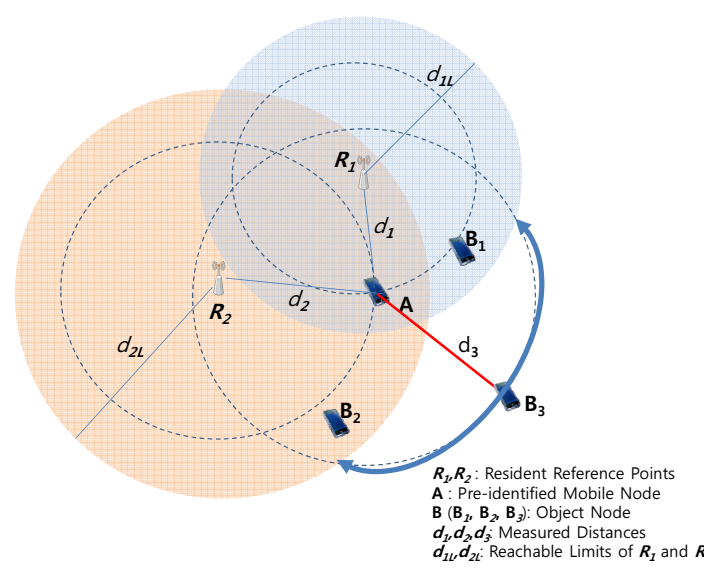

Fig. 3. Locating with Mobile Coordinate

\section{PROPOSED ALGORITHM}

\section{A. System Model}

As shown in Fig. 4, in $x-y$ coordinate, we make the angle between the measured orientation of the object and the $x$ coordinate as the movement direction angle. In this study we introduce three such parameters as the predicted orientation of last moment, the measured orientation of current moment, and the predicted orientation of current moment. The movement direction angle is used as the system state. 


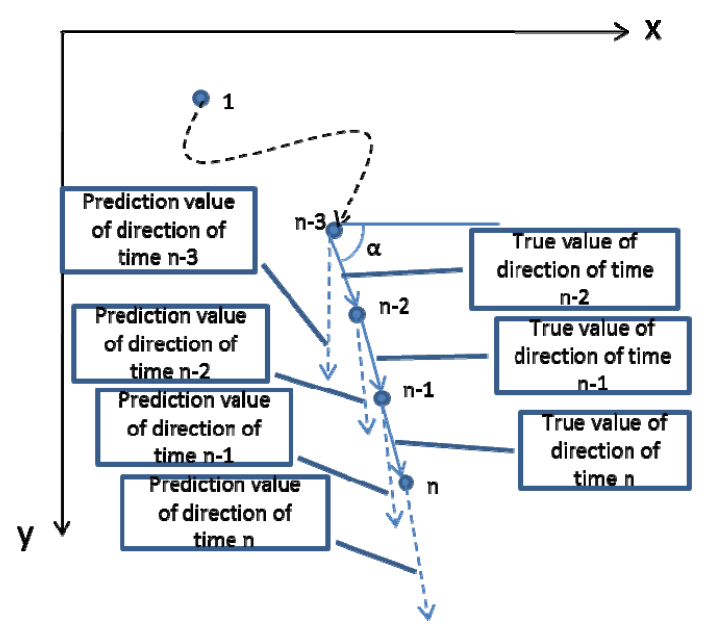

Fig. 4. Movement direction angle

\section{B. PID-controlled Moving Objects Spatio-Temporal Model Algorithm}

In a moving-average model of Moving Object SpatioTemporal (MOST)[10] the scalar part of the prediction speed of time $n+1$ is expressed as following:

$$
|\vec{v}|=\frac{1}{p} \sum_{i-1}^{p} v_{n-1},
$$

where $v$ is the speed of a target object at time $n$, and $p$ is the means of its speed in all previous time. The direction part of the prediction speed of time $n+1$ is equal to the one of the target speed at time $n-1$.

The goal is to make Prediction Value = True Value. True Value is a true value of direction of time $n$, while Prediction Value is a prediction value of direction of time $n$. There is no mechanism to reduce an error between True Value and Prediction Value. So we want to work out a mechanism to minimize the error. Since the PID controller is generally acceptable for optimization in automatic control systems, we choose it as a mechanism to minimize the error[11].
The proposed PID-controlled Moving Objects SpatioTemporal Model Algorithm can be considered as two parts: controlling part and controlled part. The controlling part is a PID controller. The controlled part is a Moving Objects Spatio-Temporal model algorithm. The input is True Value of direction of time $n$, and the output is Prediction Value of direction of time $n$, supposing the current time is time $n$. The feedback is Prediction Value of the direction of the time $n-1$. Setpoint is the expected value of the error between Prediction Value of the direction of the time $n-1$ and True Value of the direction of the time $n$. Obviously, setpoint is $0 . A$ is the error between Prediction Value of the direction of the time $n-1$ and True value of the direction of the time $n$. The error is a difference between $A$ and setpoint of the time $n$.

$$
\begin{aligned}
\text { PID Value } & =P \text { Value }+I \text { Value }+D \text { Value } \\
& =K_{p} e(t)+K_{i} \int_{0}^{\tau} e(\tau) d \tau+K_{d} \frac{d e(t)}{d t},
\end{aligned}
$$

where $e(\tau)$ is the error. $K_{p}, K_{i}$ and $K_{d}$ are the coefficients of $P$ Value, I Value, and $D$ Value respectively.

Thus the whole expression of the PID-controlled Moving Objects Spatio-Temporal Model is as following:

$$
\text { Prediction Value }(t)=\text { True Value }(t)-P I D \text { Value }(t)
$$

$$
\begin{aligned}
& \text { PID Value }=K_{p} e(t)+K_{i} \int_{b}^{\tau} e(\tau) d \tau+K_{d} \frac{d e(t)}{d t} \text { (4) } \\
& e(t)=\text { True Value }(t)-\operatorname{Pr} \text { ediction Value }(t-1)(5)
\end{aligned}
$$

Fig. 5 shows a conceptual diagram of the PIDcontrolled Moving Objects Spatio-Temporal Model proposed in this paper. In this paper, we choose $K_{p}=0.1$, $K_{i}=0.005$, and $K_{d}=0$.

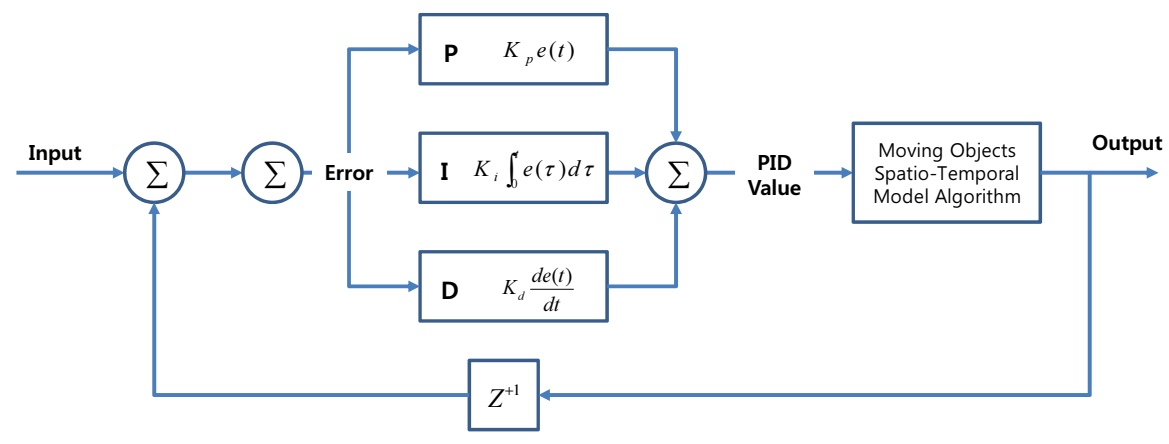

Fig. 5. Proposed PID-controlled Moving Objects Spatio-Temporal Model 


\section{SIMULATION RESULTS}

Simulation results compare the True direct (TRUE_A) with five predictive directions of every point of Target A. Those predictive directions were calculated by the Recursive Least Squares algorithm (Least A), the Kalman Filter estimation algorithm (Kalman_A), the Stochastic Approximation algorithm (Stochasic_A), the Moving Object Spatio-Temporal Model Algorithm (Model_A), and the proposed PID-controlled MOST Model algorithm (Testalgor_A).

Fig. 6 shows to compare the true direction with five predictive directions of every point of Target $\mathrm{A}$ in each step. Calculated differences between true direction and predictive one of each algorithm are

$$
\begin{aligned}
& \sum \mid \text { Diff_Least_A } \mid=502.74149, \\
& \sum \mid \text { Diff_Kalman_A } \mid=451.844292, \\
& \sum \mid \text { Diff_Stochastic_A } A=485.14598, \\
& \sum \mid \text { Diff_Model_A }=337.151869, \text { and } \\
& \sum \mid \text { Diff_Testalgor_A } \mid=333.661331 .
\end{aligned}
$$

From these, it can be observed that the case of Testalgor A, i.e. the proposed algorithm, is the closest to the true value. This is similar to result of Diff_Model_A.

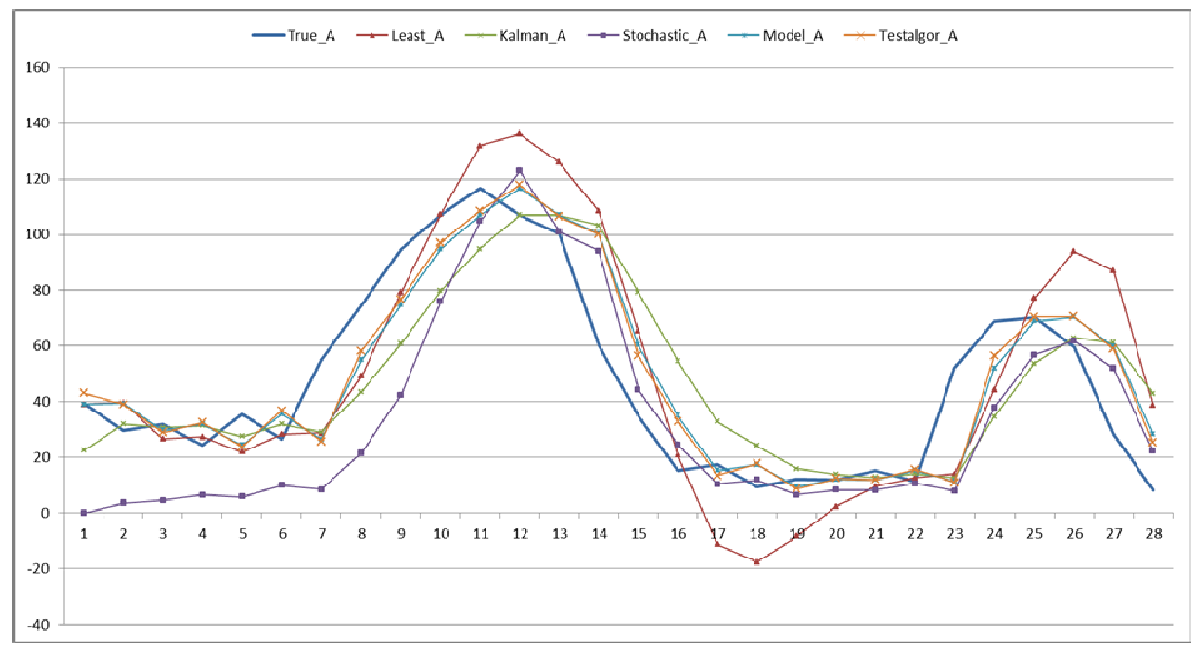

Fig. 6. Simulation results: estimated directions

Fig. 7 shows the differences between the true direction and each of five predictive directions, which is re-shaped from the same result as shown in Fig. 6. The graph of TRUE_A displays always zero. For more comprehensive simulation results, cumulative difference values for each of five predictive directions are shown in Fig. 8. It is obvious that Testalgor_A, i.e. the proposed algorithm, makes less difference values than any other algorithms according as getting more number of simulation steps.

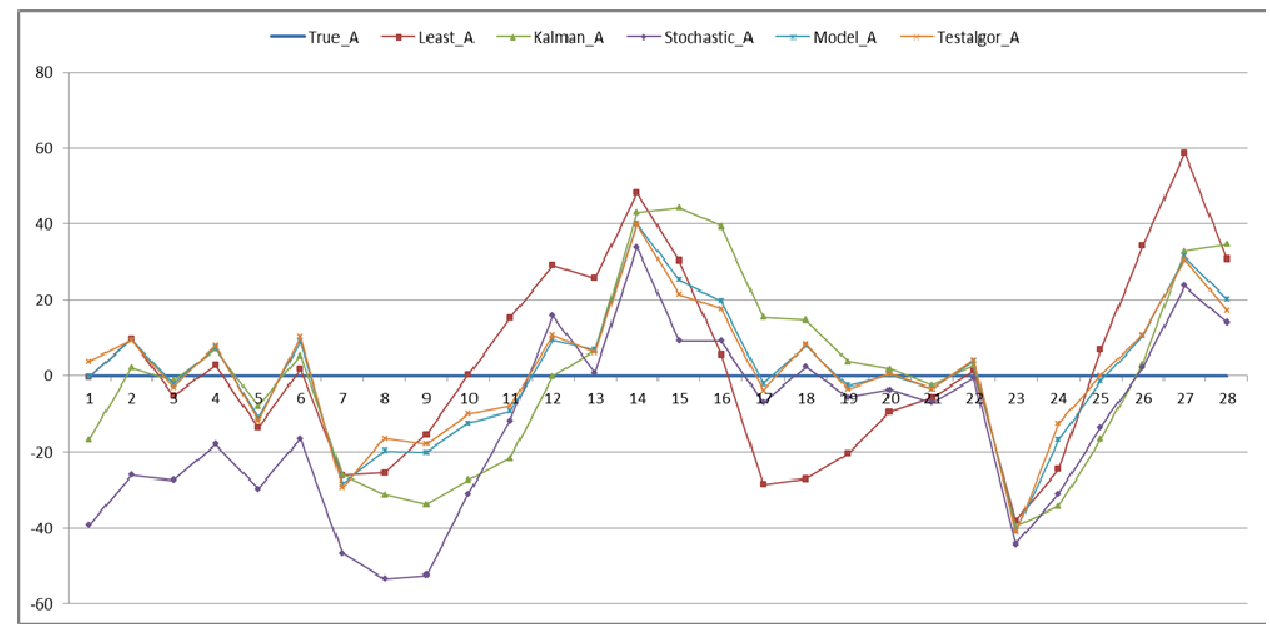

Fig. 7. Simulation results: differences between true direction and each of five predictive directions 


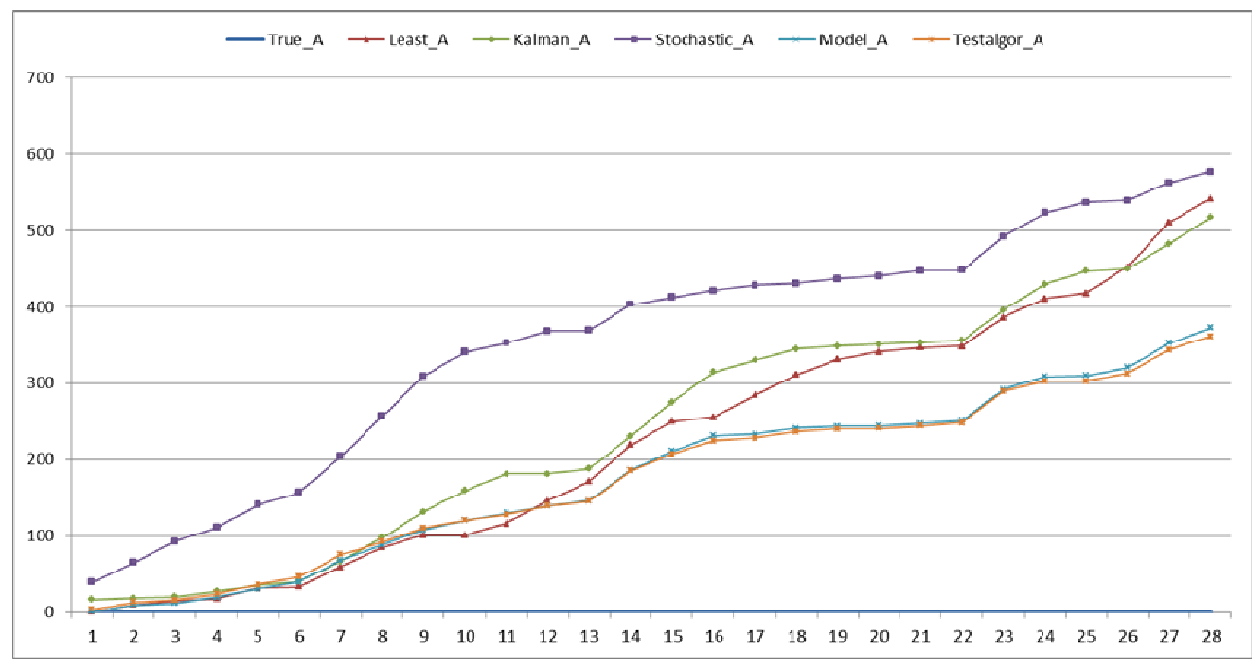

Fig. 8. Simulation results: cumulative differences

Since the difference between predictive direction and true direction is $e(t) \in(-180,180)$ in theory, it becomes $K_{p} e(t) \in(-18,18)$. Supposing the current time is 28 which means the system has got target's situation for 28 times,

$$
K_{i} \int_{0} e(t) d t \in(-25.2,25.2)
$$

and,

$$
\begin{aligned}
& \text { PID Value }=P_{p} e(t)+K_{i} \int_{0} e(t) d t \in(-25.2,25.2), \\
& \text { PID Value } \in(-43.2,43.2) .
\end{aligned}
$$

The output of new algorithm Prediction valueNew $(t)$ is equal to the output of the MOST algorithm Prediction valueMOST $(t)$ minus PID Value $(t)$, i.e.

$$
\begin{array}{r}
\text { Prediction valueNew }(t)=\text { Prediction valueMOST }(t)- \\
\text { PID Value, PID Value } \in(-43.2,43.2) .
\end{array}
$$

But in simulation environment, the difference between predictive direction and true direction $e(t) \in(-80,80)$, so $K_{p} e(t) \in(-8,8)$. Supposing current time is 28 ,

$$
K_{i} \int_{0} e(t) d t \in(-4.9,4.9)
$$

and,

$$
\begin{aligned}
& \text { PID Value }=P_{p} e(t)+K_{i} \int_{0}^{t} e(t) d t \in(-25.2,25.2), \\
& \text { PID Value } \in(-12.9,12.9) .
\end{aligned}
$$

Prediction valueNew $(t)=$ Prediction valueMOST $(t)-$ PID Value, PID Value $\in(-12.9,12.9)$. (11)

The new algorithm is thus similar to the MOST one.

\section{CONCLUSIONS}

This paper presents a method of identifying locations of mobile objects in real-time, which is applicable even to the case that an object node could not get in touch with all three base stations. A predictive method, called PIDcontrolled Moving Objects Spatio-Temporal Model Algorithm, is proposed for the basis of this method. Simulation results verified that not only the presented method can be validly enough for applying even to the cases of partial reachability but also the proposed algorithm can outperforms comparatively.

Although using the PID controller in the proposed algorithm makes the result closed to the true value, the result is similar to the original MOST algorithm. The further work is modifying parameters of the PID controller or selecting a better control mechanism in order to achieve better results.

\section{REFERENCES}

[1] Grimm, R. Edelhausser, T Kokai, G., "An Efficient Configuration Method for Real Time Locating Systems," Proc. of the 2nd International Conf. on Logistics and Industrial Informatics, pp.1-6, 2009.

[2] Yi Jiang, et al., "An asymmetric double sided two-way ranging for crystal offset," Proc. of the Int. Symposium on signals, systems and Electronics, IEEE, pp. 525-528, 2007.

[3] Y. Jiang, et al., "An Asymmetric Double Sided Two-Way Ranging for Crystal Offset," Proc. of International Symposium on Signals, Systems and Electronics (ISSSE'07), pp. 525 528, July 2007.

[4] H. Cho, et al., "Real Time Locating System for Wireless Network using IEEE 802.25.4 Radio," Proc. of Sensor, Mesh and Ad Hoc Communications and Networks(SECON), pp. 578 - 580, June 2008.

[5] Jeffrey Hightower, et al., "A Survey and Taxonomy of Location Systems for Ubiquitous Computing," IEEE Computer, vol. 34, no. 8, pp. 57-66, August 2001.

[6] Bin Ko, et al., "Data Transport Performance in Various and Interworked u-Health Service Networks," Proc. of KICS Autumn Conference 2008, Seoul, Nov. 2008(In Korean). 
[7] Kyou H. Lee, et al., "A Study on Improving Performance of the Network Transport by Interworking Different Protocols," Journal of Maritime Information and Communication Sciences (KIMICS), Vol.13, No.1, Jan. 2008 (In Korean).

[8] Kyou H. Lee, "Network Accessibility and Data Transport Performance of Interworking Mobile Ad Hoc Networks," International Journal of Maritime Information and Communication Sciences (IJMICS), Vol.7, No.1, December 2009.

[9] Kyou H. Lee, et al., "Real-time Locating of Mobile Objects Based on References of Identified Mobile Nodes and Resident Coordinates," Proc. of 2010 Int'l Conf. on Korea Institute of Maritime Information \& Communication Sciences (ICKIMICS2010), Gold Coast, Australia, July 2010.

[10] He Yunbin, Fan Shoudem, Hao Zhongxiao "Whole Trajectory Modeling of Moving Objects Based on MOST Model," Computer Engineering, Vol. 34 No. 16, August 2008.

[11] http://en.wikipedia.org/wiki/PID_controller

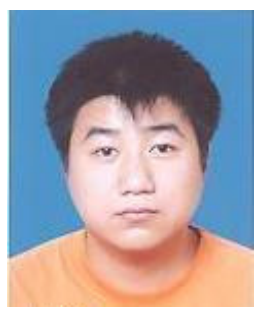

Wang Zhi received his B.S. degrees from Huazhong University of Science and Technology. He is studying in Inje University, Gimhae, Korea, for M.S degree in Department of Electronic Telecommunication Engineering. His current research interests include ubiquitous networks, embedded systems, smart home network.

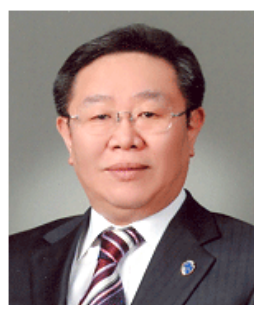

Kil Young Sung received the B.S. degree in Electronics Engineering from Kyeongbuk National University, Daegu, Korea, in 1980 and the M.S. degree in Electronics Engineering from Kunkuk University, Seoul, Korea, in 1985, respectively. He received the $\mathrm{Ph}$. D degree in Electronics Engineering from Pukyong National University, Pusan, Korea, in 2000. He is a professor of department of Information and Communication Engineering, Gyeongsang National University, Korea. His research interests include VLSI array, computer architecture, and image compression.

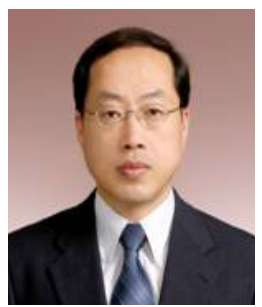

Kyou Ho Lee received his B.S and M.S degrees from Kyeongbuk National University Daegu, Korea, all in electronics engineering in 1980 and 1982, respectively, and his Ph.D degree from the University of Gent, Belgium, in information and computer engineering in 1998. From 1983 to 2005 , he had been a team leader and principal member of research staff in ETRI, Daejeon, Korea. From 1986 to 1988, he also worked as a researcher with AIT Inc., San Jose, CA, USA. Since 2005, he joined Inje University, Gimhae, Korea, as a full professor in Department of Information and Communications Engineering. His current research interests include embedded and cyber physical systems, high speed and ubiquitous network, smart home, etc. 